\title{
Effect of Cation Distribution on Structural and Magnetic Properties of Nickel Cobalt Zinc Ferrites
}

\author{
S. J. Azhagushanmugam, ${ }^{1}$ N. Suriyanarayanan, ${ }^{2}$ and R. Jayaprakash ${ }^{3}$ \\ ${ }^{1}$ Department of Physics, Muthayammal Engineering College, Rasipuram, Tamil Nadu 637 408, India \\ ${ }^{2}$ Department of Physics, Government College of Technology, Coimbatore, Tamil Nadu 641 013, India \\ ${ }^{3}$ Department of Physics, SRMV College of Arts and Science, Coimbatore, Tamil Nadu 641 020, India
}

Correspondence should be addressed to S. J. Azhagushanmugam; azhagushanmugam@yahoo.co.in

Received 1 June 2013; Accepted 8 September 2013

Academic Editor: Yong Ding

Copyright (C) 2013 S. J. Azhagushanmugam et al. This is an open access article distributed under the Creative Commons Attribution License, which permits unrestricted use, distribution, and reproduction in any medium, provided the original work is properly cited.

\begin{abstract}
Nanoparticles of $\mathrm{Ni}_{(0.8-x)} \mathrm{Co}_{(0.2)} \mathrm{Zn}_{(x)} \mathrm{Fe}_{2} \mathrm{O}_{4}(x=0.2,0.4$, and 0.6) are prepared by chemical coprecipitation method. Effects of zinc substitution on structural and magnetic properties have been investigated. The X-ray diffraction and infrared spectroscopy are used to characterize the samples. The XRD pattern of the samples provides evidence of single phase formation of spinel structure with cubic symmetry. It is observed that the particle size decreases and lattice parameter increases with the increase in zinc concentration. TEM micrographs show a well-defined nanocrystalline state with an average particle size of around $\approx 17 \mathrm{~nm}$. The B-H loops of all samples that are obtained by using vibrating sample magnetometer are displayed. The effect of $\mathrm{Zn}$ addition on saturation magnetization and the coercivity of all the samples are discussed.
\end{abstract}

\section{Introduction}

$\mathrm{Co}-\mathrm{Zn}$ ferrites are used for magnetic applications such as transformer core, noise filters, and recording heads because of their excellent magnetic properties such as high initial permeability and high saturation magnetization. However these ferrites have certain limitation for magnetic applications at higher frequencies. Although Ni-Zn ferrites have a wide spread role in many technological applications due to their low eddy currents and dielectric losses, inferior magnetic properties at higher frequencies limit their use. Properties of these ferrites (Co-Zn and $\mathrm{Ni}-\mathrm{Zn}$ ) have been investigated extensively $[1,2]$; however, a very few reports are available on the combination of these two [3]. In a previous work, the authors have reported [3] the effects of zinc substitution on structural and magnetic properties of $\mathrm{Ni}-\mathrm{Zn}$-Co ferrite. In this work, an attempt has been made to understand the effect of $\mathrm{Zn}$ substitution on structural and magnetic properties of $\mathrm{Ni}_{(0.8-x)} \mathrm{Co}_{(0.2)} \mathrm{Zn}_{(x)} \mathrm{Fe}_{2} \mathrm{O}_{4}(\mathrm{NCZF}$ ) (where $x=0.2$, 0.4, and $0.6)$. Nano size ferrites can be prepared by various methods including glass ceramic method, hydrothermal method, ultrasonic cavitation approach, mechanical milling, sol-gel method, and coprecipitation method. In the present work, nanoparticles of NCZF are prepared by the coprecipitation method, which had been previously reported as a good method for controlling the stoichiometric compositions and reducing the pollutions since no organic substances are involved during the preparation [4].

\section{Experimental}

2.1. Synthesis. Nanoparticles of NCZF were prepared by chemical coprecipitation route. In order to obtain the desired compositions, stoichiometric amounts of $\mathrm{NiCl}_{2} \cdot 6 \mathrm{H}_{2} \mathrm{O}$, $\mathrm{CoCl}_{2} \cdot 6 \mathrm{H}_{2} \mathrm{O}, \mathrm{ZnCl}_{2} \cdot 6 \mathrm{H}_{2} \mathrm{O}$, and $\mathrm{FeCl}_{3} \cdot 6 \mathrm{H}_{2} \mathrm{O}$ were dissolved in ultrapure water. The neutralization was carried out with sodium hydroxide solution. The reaction temperature was kept at $60^{\circ} \mathrm{C}$ for $15 \mathrm{~min}$. The $\mathrm{pH}$ of the reaction was kept at 12. The precipitates were thoroughly washed with distilled water until they were free from sodium and chloride ions. The product was dried in an electric oven at a temperature of $100^{\circ} \mathrm{C}$ to remove water contents. The dried powder was grained well in a cleaned agate mortar and pestle. By using high temperature muffle furnace, the grained powders were sintered at $600^{\circ} \mathrm{C}$ for $2 \mathrm{hrs}$, then the furnace was switched 
TABLE 1: Particle size, lattice parameter, and X-ray density of nickel cobalt zinc ferrite.

\begin{tabular}{lccc}
\hline $\begin{array}{l}\text { Composition } \\
(x)\end{array}$ & $\begin{array}{c}\text { Particle size } \\
(\mathrm{nm})\end{array}$ & $\begin{array}{c}\text { Lattice } \\
\text { parameter }(\AA)\end{array}$ & $\begin{array}{c}\text { X-ray density } \\
\left(\mathrm{gm} / \mathrm{cm}^{3}\right)\end{array}$ \\
\hline 0.2 & 62.49 & 8.231 & 8.572 \\
0.4 & 60.46 & 8.236 & 8.556 \\
0.6 & 17.84 & 8.245 & 8.528 \\
\hline
\end{tabular}

off. The samples were cooled by slow cooling to room temperature.

2.2. Characterization. The structural and crystallite sizes were analyzed by X-ray diffraction (Rigaku ultima III model) using CuK $\alpha(\lambda=1.5406 \AA)$ radiation. The average crystallite sizes of the samples estimated from the line width of the high intense peaks, lattice constant $(a)$, and X-ray density $\left(\rho_{x}\right)$ were calculated using well-known mathematical relations $[5$, 6]. TEM micrographs were recorded by using transmission electron microscope (Philips model CM 200) to investigate powder morphology as well as grain size. Room temperature magnetization and the hysteresis loops were recorded by means of a parallel field vibrating sample magnetometer (Lakeshore VSM 7410 model). The IR spectra were recorded by using Perkin-Elmer FTIR RXI spectrometer in the frequency range $400-4000 \mathrm{~cm}^{-1}$.

\section{Result and Discussion}

3.1. XRD Analysis. The XRD pattern for the samples of NCZF sintered at $600^{\circ} \mathrm{C}$ is shown in Figure 1. Analysis of the diffraction pattern of all the samples confirmed the formation of spinel cubic structure. This was also confirmed by two absorption peaks at frequencies $\approx 409.64 \mathrm{~cm}^{-1}$ and $\approx 579.68 \mathrm{~cm}^{-1}$ in FTIR spectra [7]. The average crystallite size for each sample was calculated using the Scherer formula [8] $t=0.9 \times \lambda / \beta \cos \theta$, where $t$ is the particle size, $\lambda$ is the wave length of X-ray, $\beta$ is the full width half maximum, and $\theta$ is the angle of diffraction. The values of X-ray density of each sample were calculated by using the formula $D_{x}=8 \mathrm{M} / \mathrm{Na}^{3}$, where $D_{x}$ is X-ray density, $M$ is the molecular weight, $N$ is Avogadro's number, and $a$ is the lattice parameter. The values of particle size, lattice parameter, and X-ray density of the synthesized samples are tabulated in Table 1.

The variations of particle size and lattice parameter with an increase in Zinc concentration are shown in Figures 2 and 3. From Figure 2, it is seen that the particle size of samples was decreased with the increase in $\mathrm{Zn}$ concentration. The above observation tells us that presence of Zinc obstructs the crystal growth [1]. This is due to the increase in crystal surface temperature with an addition of zinc, decreasing the molecular concentration at the crystal surface. From Figure 3, it is seen that the lattice parameter increases with $\mathrm{Zn}$ concentration. This increase of lattice parameter can be easily explained on the basis of ionic radii differences of the constituent ions, $\mathrm{Zn}^{2+}(0.83 \AA), \mathrm{Ni}^{2+}(0.78 \AA), \mathrm{Co}^{2+}(0.72 \AA)$, and $\mathrm{Fe}^{3+}(0.67 \AA)$. The increase in lattice parameter with

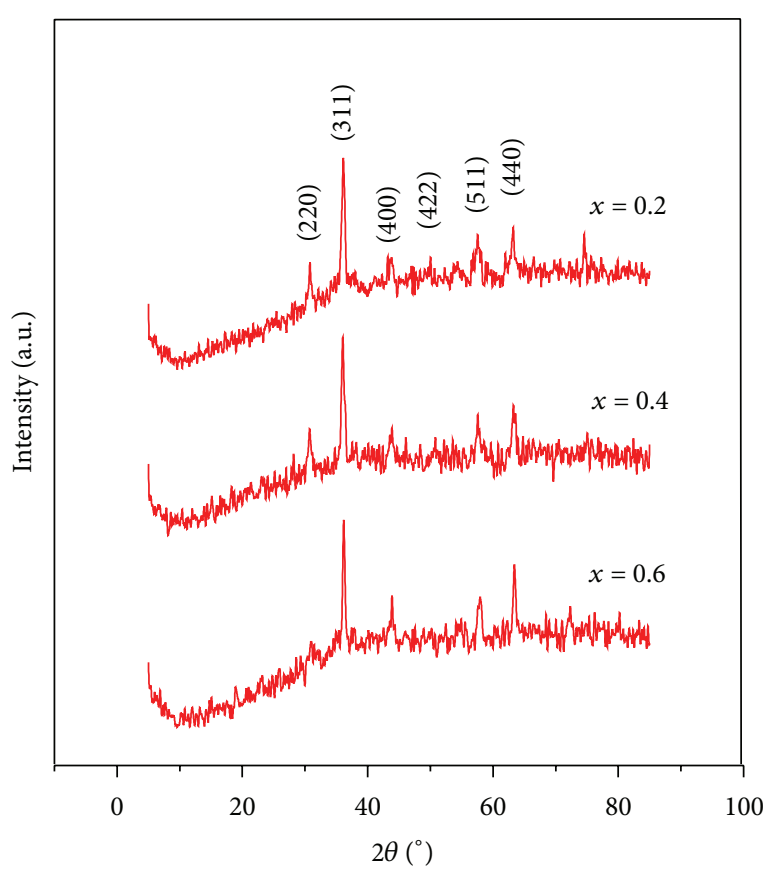

FIGURE 1: XRD patterns of nickel cobalt zinc ferrite.

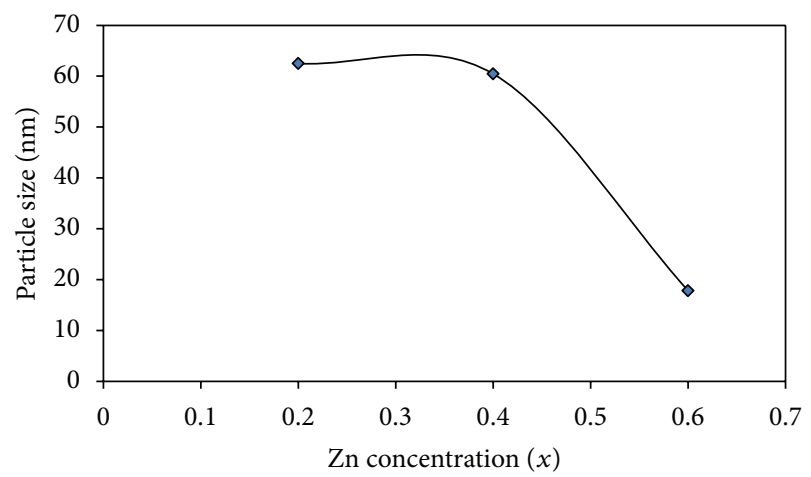

Figure 2: Variation of particle size with $\mathrm{Zn}$ in NCZF.

addition of $\mathrm{Zn}^{2+}$ is due to the fact that its ionic radius is greater than other component ions for which it substitutes. Similar observations had been reported by other researchers [9].

3.2. FTIR Analysis. The IR absorption spectra recorded in the range $4000-400 \mathrm{~cm}^{-1}$ are shown in Figure 4 for NCZF samples. The two prominent absorption bands, approximately in the ranges $\approx 500$ and $\approx 400 \mathrm{~cm}^{-1}$, are caused by stretching vibrations of tetrahedral metal ion and the presence of divalent metal ion-oxygen complexes in the octahedral sites [10, 11]. From the Figure 4, it is also observed that the absorption bands in the frequency ranges $\approx 500$ and $\approx 400 \mathrm{~cm}^{-1}$ shift gradually towards the low frequency side with an increase in $\mathrm{Zn}$ concentration. This may be due to the increase in the bond length as a consequence of the addition of larger $\mathrm{Zn}$ ions. Similar results had been also observed by Shaikh et al. [10]. 


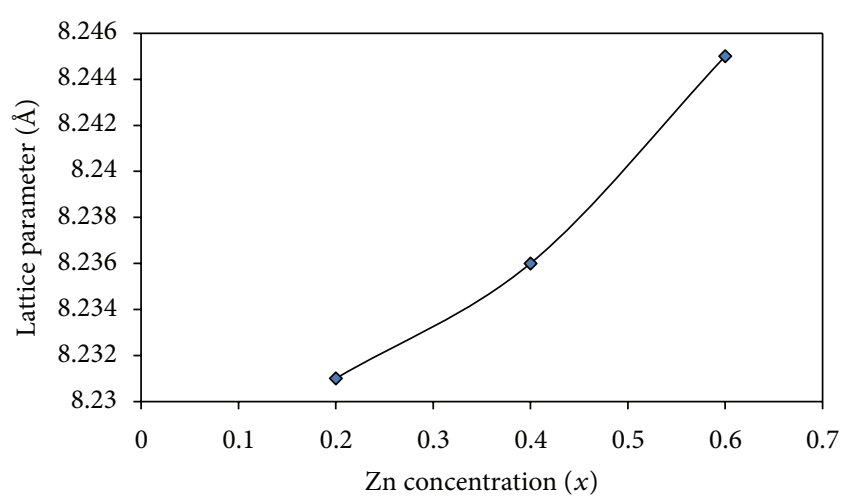

FIgURE 3: Variation of lattice parameter with $\mathrm{Zn}$ in NCZF.

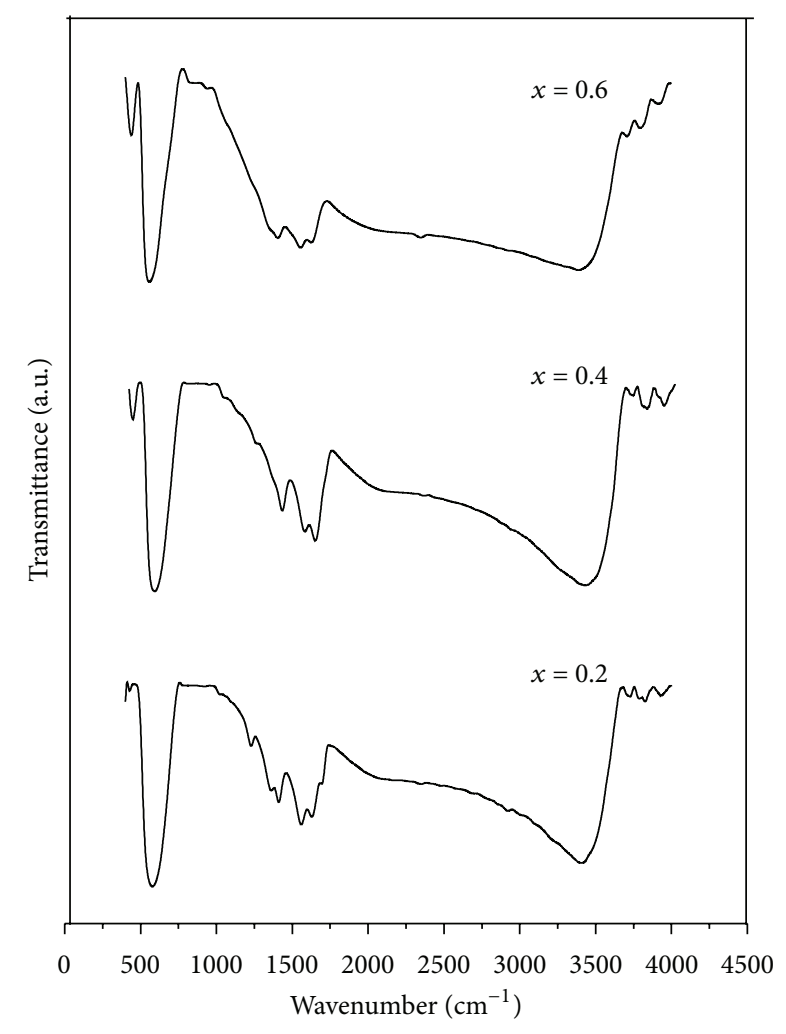

FIgURE 4: FTIR spectrum of NCZF.

3.3. VSM Analysis. The room temperature B-H hysteresis loops of NCZF compositions sintered at $600^{\circ} \mathrm{C}$ are shown in Figure 5. The loops areas are minimal with small coercivities indicating that the hysteresis loop is negligible in these ferrites. The magnetic properties such as saturation magnetization $\left(M_{s}\right)$, coercivity $\left(H_{c}\right)$, retentivity $\left(M_{r}\right)$, and squareness ratio were calculated from the hysteresis data and tabulated in Table 2.

Figure 6 shows the variation of saturation magnetization as a function of $\mathrm{Zn}$ concentration. The saturation magnetization has been found to decrease significantly with substituent's concentration throughout. In the basic composition, the zinc ion (magnetic moment $0 \mu_{\mathrm{B}}$ ) occupy tetrahedral
TABLE 2: Magnetic properties of NCZF.

\begin{tabular}{lcccc}
\hline $\begin{array}{l}\text { Composition } \\
(x)\end{array}$ & $\begin{array}{c}\text { Saturation } \\
\text { magnetization } \\
M_{s}(\mathrm{emu} / \mathrm{g})\end{array}$ & $\begin{array}{c}\text { Retentivity } \\
M_{r} \\
(\mathrm{emu} / \mathrm{g})\end{array}$ & $\begin{array}{c}H_{c} \\
(\text { Gauss })\end{array}$ & $\begin{array}{c}\text { Squareness } \\
\text { ratio }\end{array}$ \\
\hline 0.2 & 45.55 & 2.02 & 59.08 & 0.044 \\
0.4 & 32.34 & 0.254 & 11.40 & 0.007 \\
0.6 & 30.86 & $3.699 E-3$ & 0.20 & $6.165 E-6$ \\
\hline
\end{tabular}

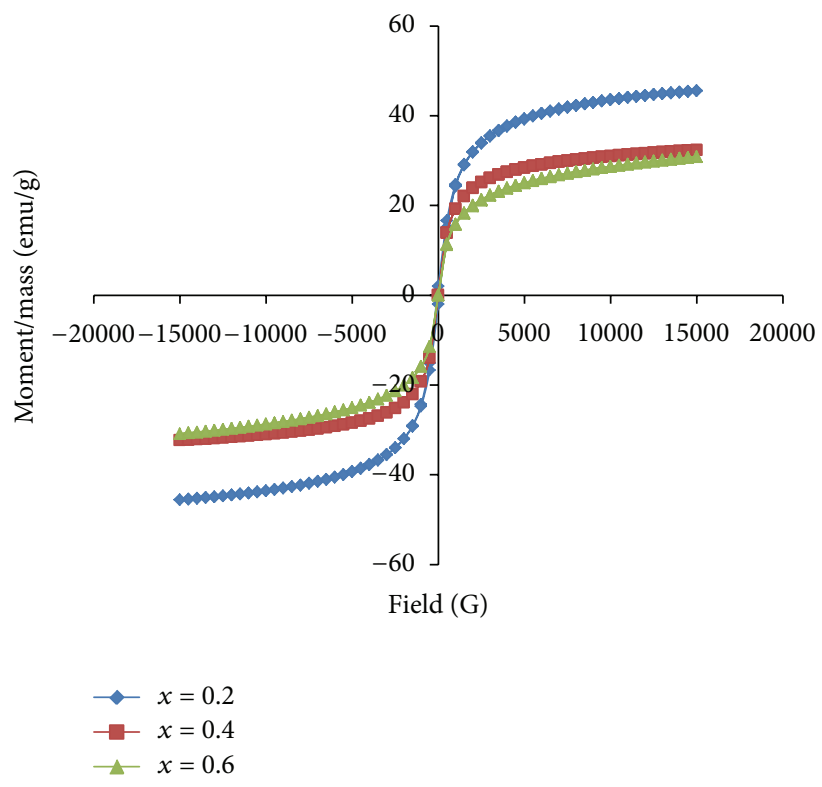

Figure 5: B-H hysteresis loops of NCZF.

(A) site. The ferric ion has magnetic moment $5 \mu_{\mathrm{B}}$ that occupies both octahedral (B) and tetrahedral (A) sites. The Ni ions have magnetic moment $2 \mu_{\mathrm{B}}$ and cobalt ion has magnetic moment $3 \mu_{\mathrm{B}}$ that occupy the octahedral $\mathrm{B}$ site. The observed variation in saturation magnetization can be explained in terms of exchange in interaction between $\mathrm{B}$ and $\mathrm{A}$ sites, respectively. The A-B interaction is known to be the strongest and dominates the $\mathrm{B}-\mathrm{B}$ and $\mathrm{A}-\mathrm{A}$ interactions.

The small quantity of ferric ion present in B site is observed to be too small to maintain the predominant A$\mathrm{B}$ exchange interaction among $\mathrm{A}$ and $\mathrm{B}$ sites. Since the paramagnetic zinc ions with magnetic moment of $0 \mu_{\mathrm{B}}$ have marked preferences in A site, substitution of zinc ions in the composition forces some ferric ions to migrate into B site. As a result of substitution, the A-B interaction as felt by the $B$ site ferric ion weakened which reduces the net magnetization [12]. From Table 2, it is also observed that because of the paramagnetic nature of the zinc ion, coercivity of $\mathrm{Ni}_{(0.8-x)}$ $\mathrm{Co}_{(0.2)} \mathrm{Zn}_{(x)} \quad \mathrm{Fe}_{2} \mathrm{O}_{4}$ samples gradually decreases with zinc concentration.

3.4. TEM Analysis. Figure 7 shows a typical transmission electron microscope (TEM) micrograph of the nanosized 


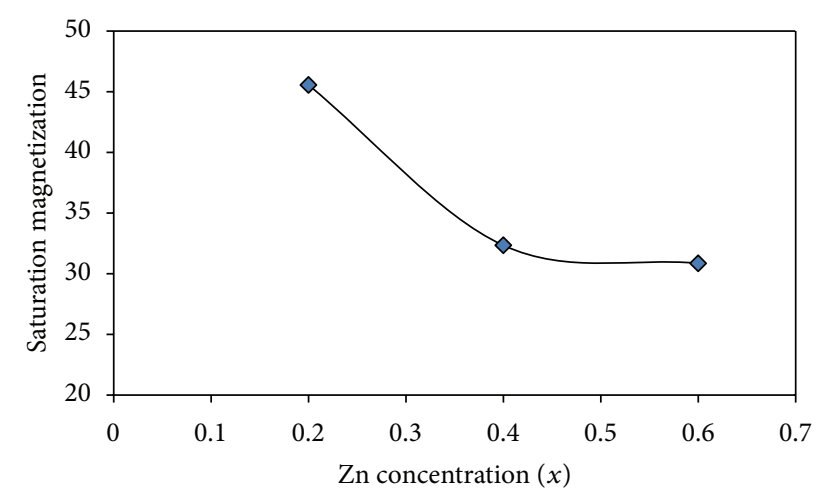

FIGURE 6: Variation of saturation magnetization with $\mathrm{Zn}$ in NCZF.

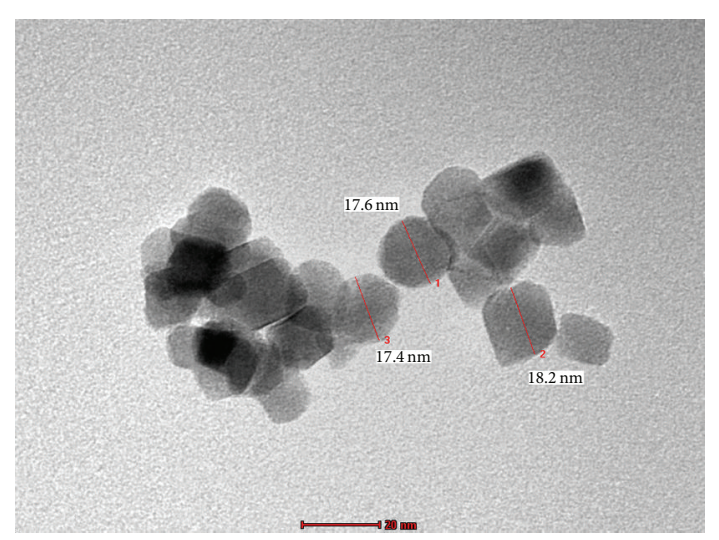

Figure 7: TEM image of NCZF.

NiCoZn ferrite with a zinc content of 0.6 . The image indicates the nanostructured nature and spherical morphology of the powder with uniform size. The particle size is about $17 \mathrm{~nm}$, which agrees well with that estimated using XRD. This reveals, as obvious from the image, that the synthesized powder is well dispersed and that no aggregates are formed.

3.5. EDX Spectra Analysis. Figure 8 shows that the compositional analysis of the nanocrystalline NCZF particles which are prepared by coprecipitation technique has been carried out using Energy-dispersive X-ray spectra. The EDX analysis reveals the presence of $\mathrm{Ni}, \mathrm{Co}, \mathrm{Zn}$, and Fe in the prepared samples. This matches well with the stoichiometry of the samples without any impurity.

\section{Conclusion}

Nanocrystalline nickel cobalt zinc particles had been synthesized by coprecipitation method. XRD patterns of the samples showed sharp peaks consistent with cubic inverse spinel structure. The particle size decreased and the lattice parameter increased with an increase in zinc concentration. TEM micrographs confirmed that the particles are in the nanometer range. The saturation magnetization and

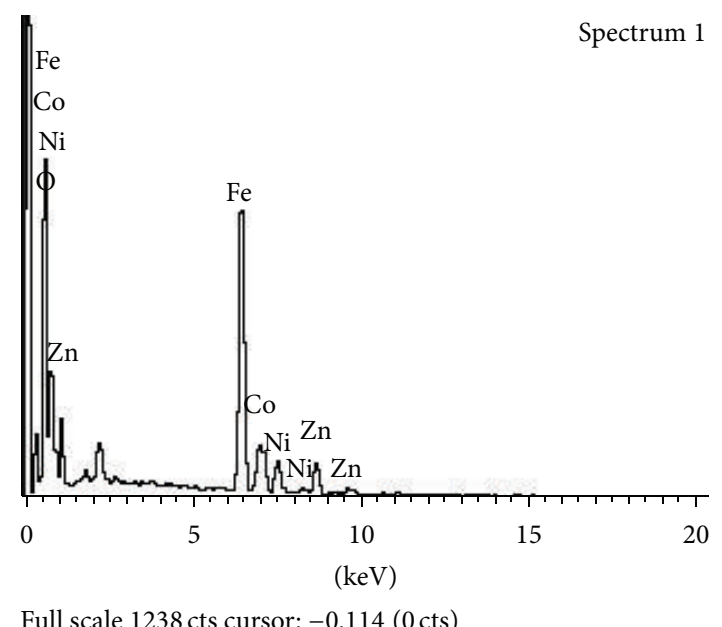

FigURE 8: EDX spectra of NCZF.

coercivity of the samples measured at room temperature are found to decrease with the increase in zinc content.

\section{References}

[1] I. H. Gul, W. Ahmed, and A. Maqsood, "Electrical and magnetic characterization of nanocrystalline $\mathrm{Ni}-\mathrm{Zn}$ ferrite synthesis by co-precipitation route," Journal of Magnetism and Magnetic Materials, vol. 320, no. 3-4, pp. 270-275, 2008.

[2] G. Vaidyanathan and S. Sendhilnathan, "Characterization of $\mathrm{Co}_{1-x} \mathrm{Zn}_{x} \mathrm{Fe}_{2} \mathrm{O}_{4}$ nanoparticles synthesized by co-precipitation method," Physica B, vol. 403, no. 13-16, pp. 2157-2167, 2008.

[3] M. M. Mallapur and B. K. Chougule, "Synthesis, characterization and magnetic properties of nanocrystalline Ni-Zn-Co ferrites," Materials Letters, vol. 64, no. 3, pp. 231-234, 2010.

[4] H.-B. Wang, J.-H. Liu, W.-F. Li et al., "Structural, dynamic magnetic and dielectric properties of $\mathrm{Ni}_{0.15} \mathrm{Cu}_{0.2} \mathrm{Zn}_{0.65} \mathrm{Fe}_{2} \mathrm{O}_{4}$ ferrite produced by $\mathrm{NaOH}$ co-precipitation method," Journal of Alloys and Compounds, vol. 461, no. 1-2, pp. 373-377, 2008.

[5] I. H. Gul, A. Z. Abbasi, F. Amin, M. Anis-ur-Rehman, and A. Maqsood, "Structural, magnetic and electrical properties of $\mathrm{Co}_{1-x} \mathrm{Zn}_{x} \mathrm{Fe}_{2} \mathrm{O}_{4}$ synthesized by co-precipitation method," Journal of Magnetism and Magnetic Materials, vol. 311, no. 2, pp. 494-499, 2007.

[6] B. D. Cullity, Elements of Xray Diffraction, Addison-Wesley Publishing Company, London, UK, 1959.

[7] U. B. Lonkar, Analysis fabrication and study of magnetically tuned overlayed/under played microstrip components [Ph.D. thesis], Shivaji University, Kolhapur, India, 2003.

[8] F. Bensebaa, F. Zavaliche, P. L'Ecuyer, R. W. Cochrane, and T. Veres, "Microwave synthesis and characterization of Co-ferrite nanoparticles," Journal of Colloid and Interface Science, vol. 277, no. 1, pp. 104-110, 2004.

[9] X. Cao, K. Sun, C. Sun, and L. Leng, "The study on microstructure and microwave-absorbing properties of lithium zinc ferrites doped with magnesium and copper," Journal of Magnetism and Magnetic Materials, vol. 321, no. 18, pp. 2896-2901, 2009.

[10] A. M. Shaikh, S. A. Jadhav, S. C. Watawe, and B. K. Chougule, "Infrared spectral studies of Zn-substituted Li-Mg ferrites," Materials Letters, vol. 44, no. 3, pp. 192-196, 2000. 
[11] D. Ravinder, "Far-infrared spectral studies of mixed lithiumzinc ferrites" Materials Letters, vol. 40, no. 5, pp. 205-208, 1999.

[12] A. K. Singh, T. C. Goel, and R. G. Mendiratta, "Effect of cation distribution on the properties of $\mathrm{Mn}_{0.2} \mathrm{Zn}_{x} \mathrm{Ni}_{0.8-x} \mathrm{Fe}_{2} \mathrm{O}_{4}$," Solid State Communications, vol. 125, no. 2, pp. 121-125, 2003. 

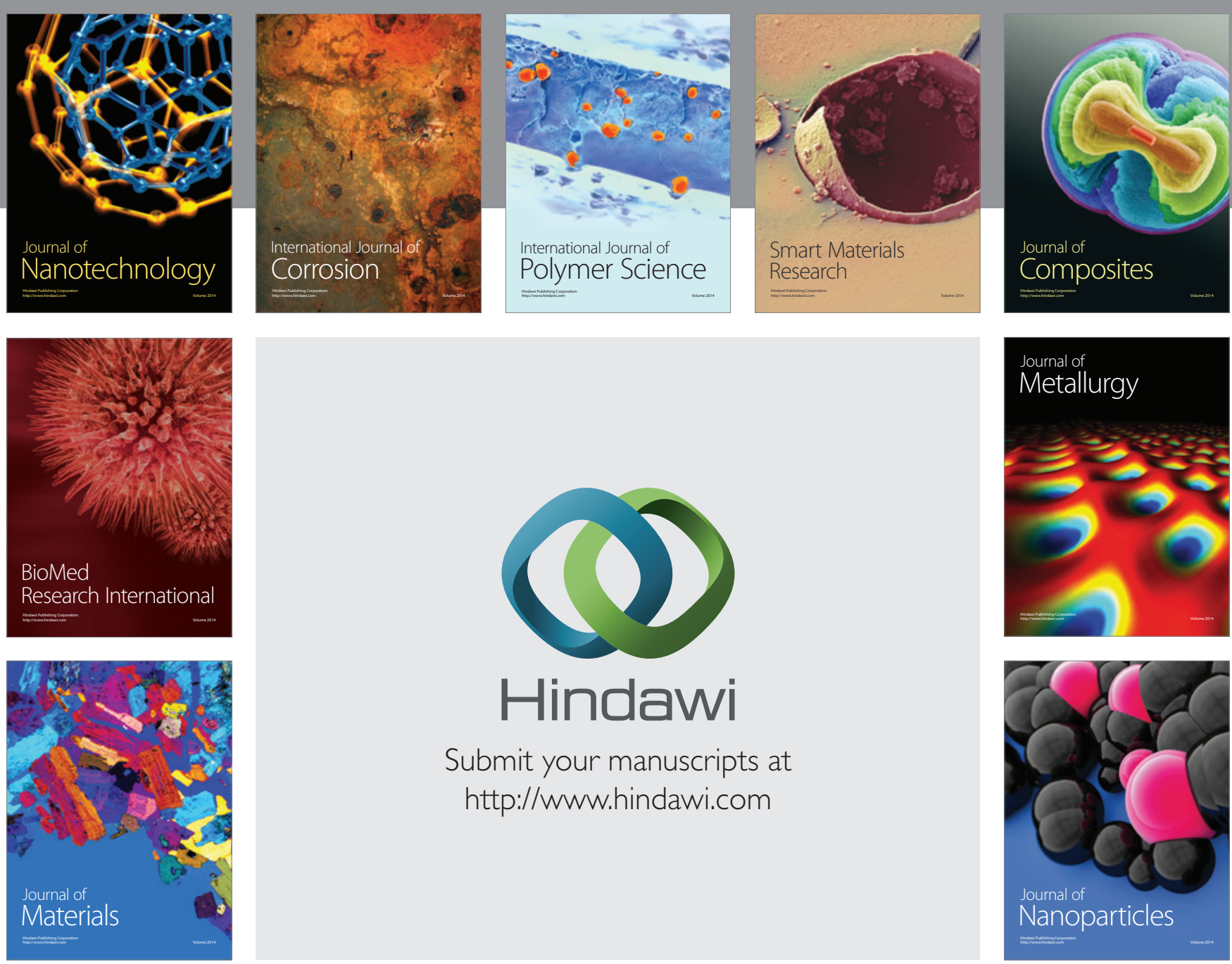

Submit your manuscripts at http://www.hindawi.com
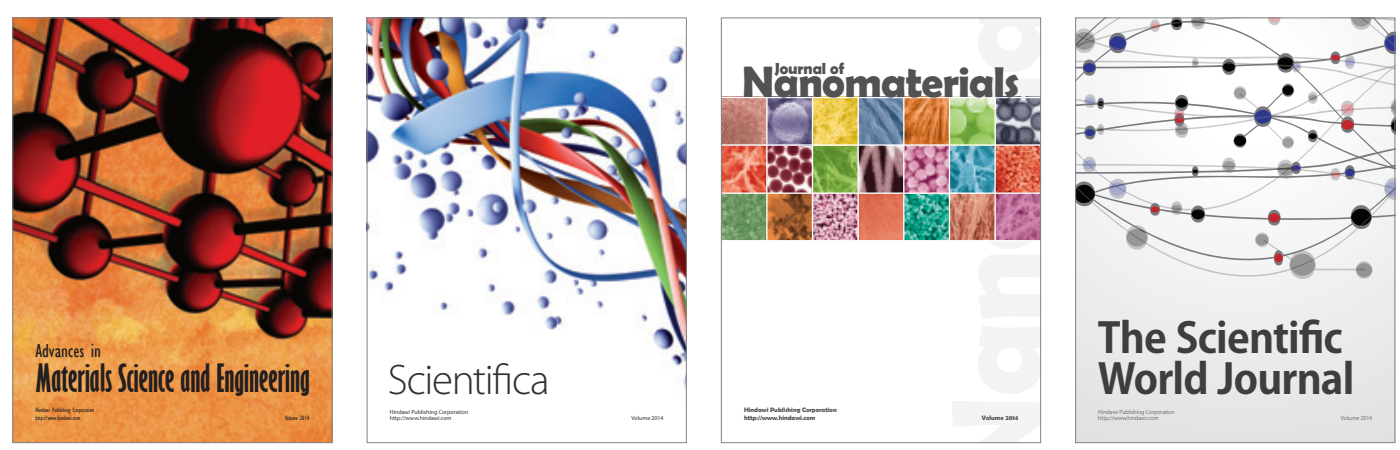

\section{The Scientific World Journal}
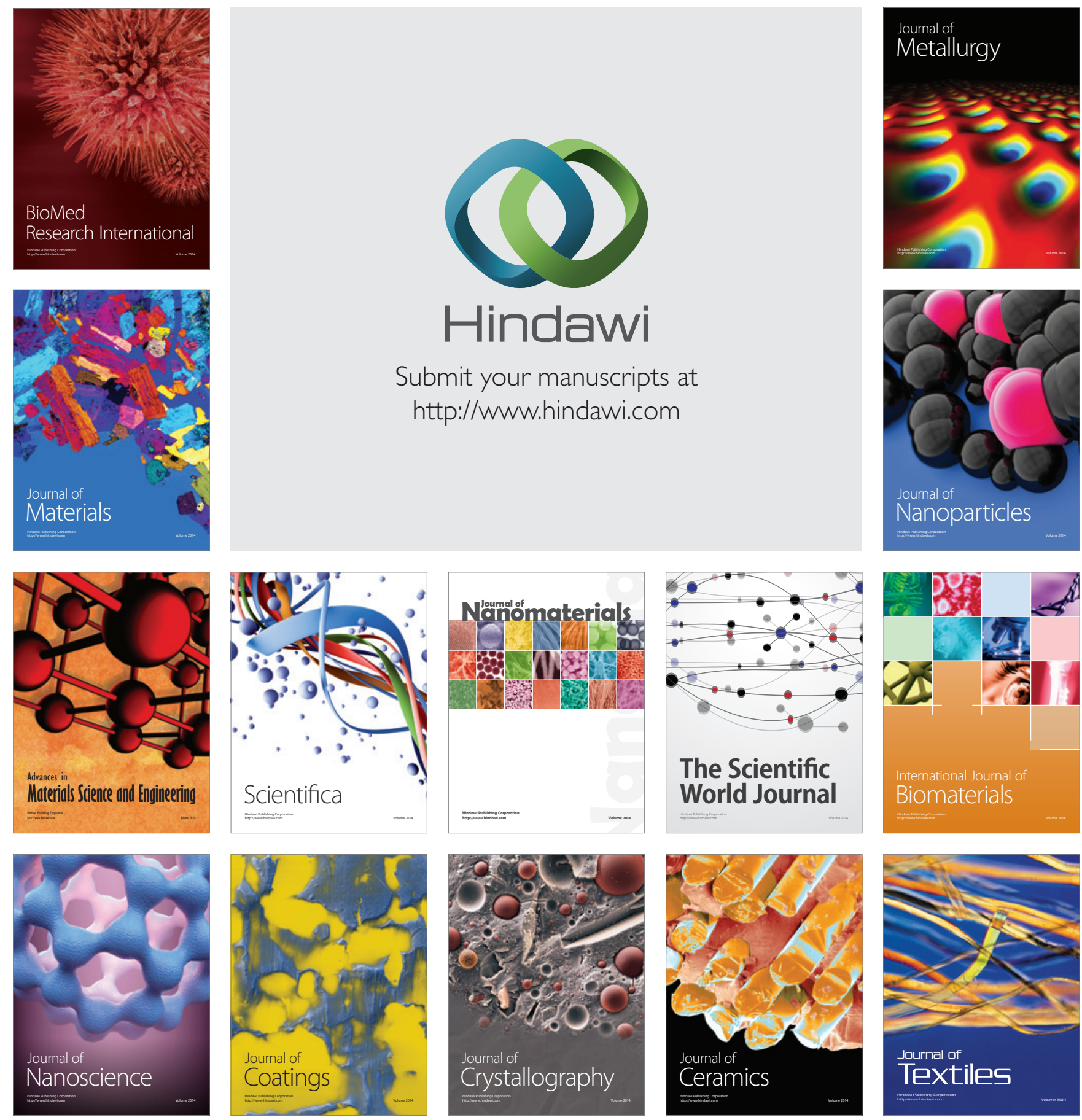